ORIGINAL ARTICLE

\title{
Molecular Docking Study of Astaxanthin Derived from Radio-Resistant Bacterium Deinococcus sp. Strain WMA-LM9 to Matrix Metalloproteinase-1, 3 (MMP-1, MMP-3)
} Wasim Sajjad, Sumra Wajid Abbasi, Liaqat Ali

\begin{abstract}
Objective: To assess role of astaxanthin in downregulation of matrix metalloproteinases (MMP-1 and MMP-3) as potential mitigator for skin aging and antioxidant for different pathological diseases.

Study Design: Cross sectional.

Place and Duration of Study: The study was carried out at Department of Biological Sciences of National University of Medical Sciences, Rawalpindi, Pakistan from June 2019 to March 2020.

Materials and Methods: The docking studies of two different matrix metalloproteinases (MMP-1, MMP-3) with astaxanthin were carried out using Autodock/vina. The structural details were obtained from protein data bank and subjected to energy minimization using the UCSF Chimera 1.12.

Results: Out of the two selected targets, we found the highest binding energy $(-11.9 \mathrm{kacl} / \mathrm{mol})$ was for stromelysin-1 and astaxanthin docked complex. Astaxanthin was found to bind within the reported, predominantly hydrophobic S1, active site of protein mostly by hydrophobic interactions. The catalytic zinc ion has also shown to establish an electrostatic interaction with histidine residues of the MMPs.

Conclusion: The current results suggest that astaxanthin has potential inhibitory activity on MMP-1 and MMP3 and can be used as treatment to sunburns and skin aging.
\end{abstract}

\section{Key Words: Antioxidants, Astaxanthin, Molecular Docking, Skin Aging, Ultra-Violet Rays.}

How to cite this: Sajjad W, Abbasi SW, Ali L. Molecular Docking Study of Astaxanthin Derived from Radio-Resistant Bacterium Deinococcus sp. Strain WMA-LM9 to Matrix Metalloproteinase-1, 3 (MMP-1, MMP-3). Life and Science. 2021; 2(1): 3-8. doi: http://doi.org/10.37185/LnS.1.1.105

This is an Open Access article distributed under the terms of the Creative Commons Attribution License (http://creativecommons.org/licenses/by/4.0), which permits unrestricted use, distribution, and reproduction in any medium, provided the original work is properly cited.

\section{Introduction}

Astaxanthin is naturally occurring xanthophyll carotenoid originally isolated from lobsters by Kuhn and Sorensen. ${ }^{1}$ It is also found in sea foods such as shrimps, crabs and fish. Astaxanthin is a highly antioxidant, anti-inflammatory and anti-cancer agent. Furthermore, it has anti-aging effects and is $y$ used in topical creams as sun blocking agent. ${ }^{2}$

Skin is the primary and largest organ of human body and provides protection against several different factors including physical, chemical and biological. ${ }^{3,4}$ Physiological and photo- aging are mechanisms of

Department of Biological Sciences

National University of Medical Sciences, Rawalpindi

Correspondence:

Dr. Wasim Sajjad

Assistant Professor, Biological Sciences

National University of Medical Sciences, Rawalpindi

E-mail: sajjadw@numspak.edu.pk

Funding Source: NIL; Conflict of Interest: NIL

Received: Mar 13, 2020; Revised: Dec 08, 2020

Accepted: Dec 20, 2020 skin aging, manifested as wrinkles, age spots and elasticity degradation. In ultraviolet radiated areas degradation of the collagen results in skin aging because of matrix metalloproteinases (MMPs). ${ }^{5}$ These are secreted from dermal fibroblasts and epidermal keratinocytes in response to ultraviolet (UV) radiations. The MMPs activation is the result of secretion of number of cytokines by reactive oxygen species in keratinocytes. ${ }^{6,7}$ Oxidative stress also results in formation of different degraded and unstable products like toxic aldehydes and ketones, protein oxidation and DNA mutations. All these results in different pathological conditions in host cells and ultimately leads to premature aging and cancer. The suppression of inflammatory cytokines and inhibition of MMPs is crucial for preventing age related skin deterioration. ${ }^{8}$

The matrix metalloproteinase group consist of metzincin super family which comprises of over 20 different zinc peptidases. ${ }^{9,10}$ This series of enzymes 
plays an important role not only in the preservation but also in turnover of some macromolecules in extracellular matrix (ECM) for example, collagen. MMP-1 is a serious initiator of many macromolecules of ECM disturbance and also involved in degradation of collagen in association with other MMPs. Different physiological factors like inflammatory cytokines, chemokine release, tumor promoter's ultraviolet radiations and other growth factors results in the expression of MMP-1 from fibroblasts in human dermal cells. The production of reactive oxygen species like $\mathrm{OH}, \mathrm{OH},{ }^{1} \mathrm{O}_{2}$ and other free radicals are early and key events in signaling processes which after exposure to ultraviolet rays ultimately leads to MMP-1 induction. ${ }^{11}$

Numerous studies have shown that astaxanthin has potential health-promoting effects in the prevention and treatment of various diseases, such as cancers, chronic inflammatory diseases, metabolic syndrome, diabetes, diabetic nephropathy, cardiovascular diseases, gastrointestinal diseases, liver diseases, neurodegenerative diseases, eye diseases, skin diseases, exercise-induced fatigue, male infertility as reviewed by Yuan. ${ }^{12}$ Astaxanthin also has a significant potential to prevent protein oxidation and breakage of DNA strands in radiation mediated cell damage. ${ }^{13}$

The major mode of action of astaxanthin is to scavenge reactive oxygen species (ROS). ${ }^{14}$ It is anticipated that the addition of astaxanthin to UVexposed fibroblasts in culture prevents the UVstimulated up-regulation of MMP-1 and MMP-3. Astaxanthin is much more effective in scavenging free radicals than other carotenoids and vitamin $\mathrm{E}$. In this study we assessed the interaction and behavior of astaxanthin with MMP-1 and MMP-3 respectively, using molecular docking approach. Furthermore, we predicted accurate binding mode of astaxanthin with matrix metalloproteinase in order to provide better insight into the down regulation of the above mentioned proteins in carcinomas.

\section{Materials and Methods}

This investigation was designed to dock the small molecule astaxanthin derived from newly isolated radio-resistant bacterium with the $3 \mathrm{D}$ structures of selected matrix metalloproteinases enzyme targets. MMPs are zinc-containing endopeptidases and collectively, these enzymes are able to degrade various components of ECM proteins. The alterations made to the ECM by MMPs may contribute in skin wrinkling, a characteristic of premature skin aging. In photo carcinogenesis, degradation of ECM is the initial step towards tumor cell invasion, to invade both the basement membrane and the surrounding stroma that mainly comprises fibrillary collagens. Additionally, MMPs are involved in angiogenesis, which promotes cancer cell growth and migration. Docking was performed by Autodock/Vina ${ }^{15,16}$, whereas docking complexes were visualized by Chimera and VMD. ${ }^{17,18}$

\section{Target Proteins Selection}

The structural details of selected MMPs, (1BQOMMP-3 and 1SU3-MMP-1), were obtained from Protein Data Bank (http://www.rcsb.org/pdb/ home). Based on their structure and substrate specificity, sixteen different types of MPPs are categorized into five main subgroups. We selected two types/subgroups namely: Stromelysins-1/MMP3 and Interstitial collagenase/MMP-1. The reason for their selection is their vital role in collagen Type-I degradation/premature skin aging (or photo aging) and skin cancers such as basal cell carcinoma (BCC), squamous cell carcinoma (SCC) (or photo carcinogenesis). Additionally, both have similar molecular masses and they are often secreted from the same cells. ${ }^{19}$

\section{Inhibitor Selection}

Astaxanthin, is reported to have protective effects against radiation-mediated cell damage $\mathrm{e}^{13}$, and therefore it has been selected for current investigation. Structure of astaxanthin is shown in fig 1.

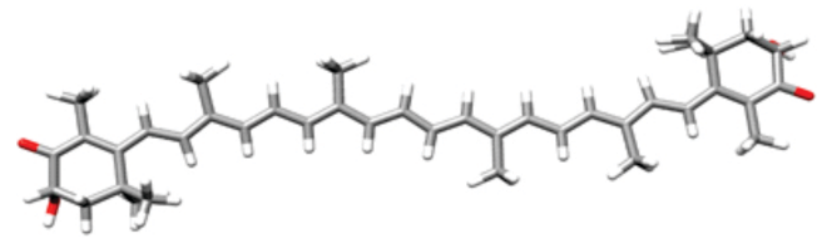

Fig 1: Structure of the of the Astaxanthin (ligand) extracted from Deinococcus sp. WM-LM9

\section{Molecular Docking by Autodock/Vina}

The crystallographic water molecules as well as Hetero Atom (HETATM) from all the selected target proteins were removed. Further proteins were subjected to energy minimization using the UCSF Chimera 1.12. Intermediary steps, such as pdbqt files 
for protein and ligands preparation and grid box creation were completed using Graphical User Interface program AutoDock Tools (ADT). ADT assigned polar hydrogens, united atom Kollman charges, solvation parameters and fragmental volumes to the protein. The zinc parameters used in this study were radius $(r=1.1 \AA)$ and well depth $(\varepsilon=$ $0.25 \mathrm{Kcal} / \mathrm{mol}) .{ }^{20} \mathrm{~A}$ charge of +2.0 e was assigned to the zinc and calcium ions in PDBQT file. AutoGrid was used for the preparation of the grid map using a grid box. ${ }^{20}$ The grid size was set to $60 \times 60 \times 60 \mathrm{xyz}$ points with grid spacing of $0.375 \AA$ and grid center was designated at dimensions ( $x, y$, and $z$ ): $-1.444,32.134$ and 45.342. A scoring grid is calculated from the ligand structure to minimize the computation time. AutoDock/Vina was employed for docking using protein and ligand information along with grid box properties in the configuration file. AutoDock/Vina employs iterated local search global optimizer. ${ }^{21}$ Although vina inherits few features from AutoDock 4 , it has shown substantial improvement in terms of time and accuracy. It also incorporated hybrid scoring function (knowledge-based as well as empirical) and a unique search algorithm. ${ }^{22}$ During the docking procedure, both the protein and ligands are considered as rigid. The results less than $1.0 \AA$ in positional root-mean square deviation (RMSD) was clustered together and represented by the result with the most favorable free energy of binding. The pose with lowest energy of binding or binding affinity was extracted and aligned with receptor structure for further analysis.

\section{Results}

Our current docking studies showed interactions between active residues and target proteins (MMP-1 and MMP-3). The lowest binding affinities from Auto Dock/Vina were used as criteria to interpret the best conformation. Out of the two selected targets, we found the highest binding energy $(-11.9 \mathrm{kacl} / \mathrm{mol})$ for stromelysin-1 and astaxanthin docked complex. Careful visual inspection of complex revealed that astaxanthin was found bound within the reported, predominantly hydrophobic $\mathrm{S} 1$, active site of protein mostly by hydrophobic interactions, making close contacts with His 201, His 205, His 211, Ser225, Phe 86, Tyr223, Asn162 and Leu164. Twelve hydrophobic bonds between carbon atoms of ligand and carbon atoms of pocket residues such as His201 (3.93 $\AA$ ),
His205 (3.93Å), His211 (3.97 ̊, $3.85 \AA ̊$ ), Ser225 (3.92

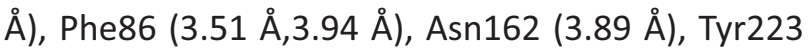

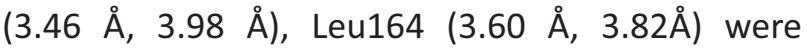
observed (Table 1; fig 2 A.2, fig 2 B).

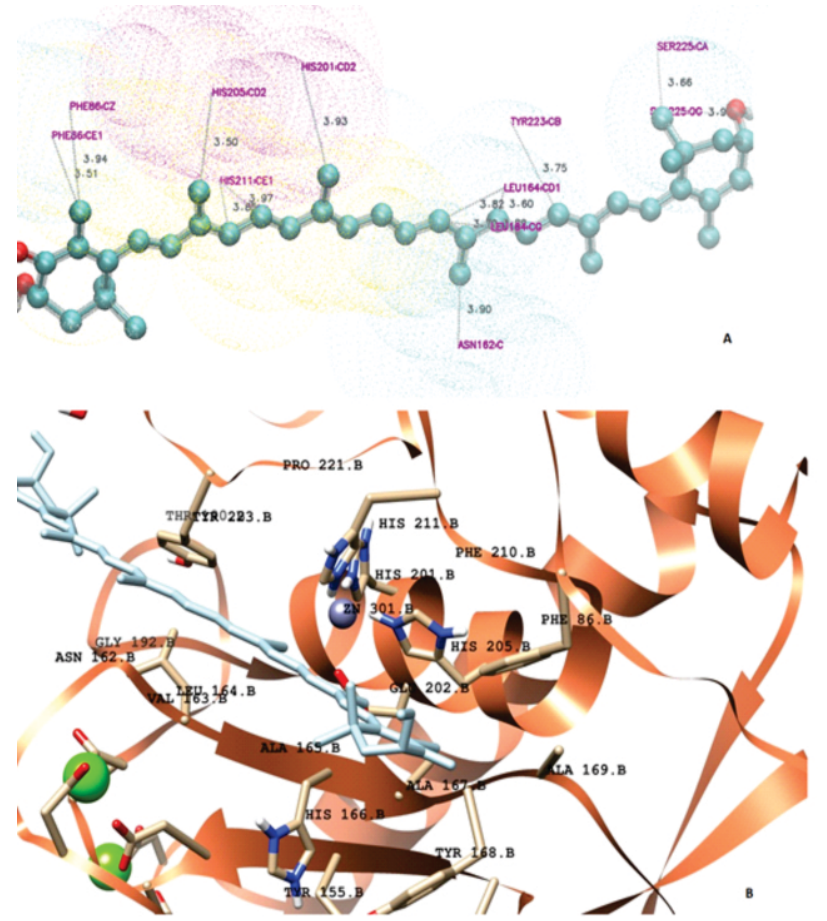

Fig 2 A. 2D depiction of stromelysin-1 and astaxanthin docked complex using VMD. 2 B: Docked pose highlighting the most active residues of protein's binding pocket Protein is in ribbons, whereas ligand is in bonds style of CHIMERAs

Additionally, catalytic zinc ions (Zn301, Zn302) also established hydrogen bond interactions with side chain atoms of three histidine residues namely His201, His205, and His211, HIS151, HIS166 and HIS179 at the distance of $1.35 \AA$, $1.28 \AA$ and $1.38 \AA$, $1.22 \AA, 1.05 \AA$ and $1.11 \AA$, respectively as shown in fig 3 , Table 1 . As the binding sites are more or less similar to reported catalytic site, thereby results suggest that astaxanthin has potential inhibitory activity on MMP-3.

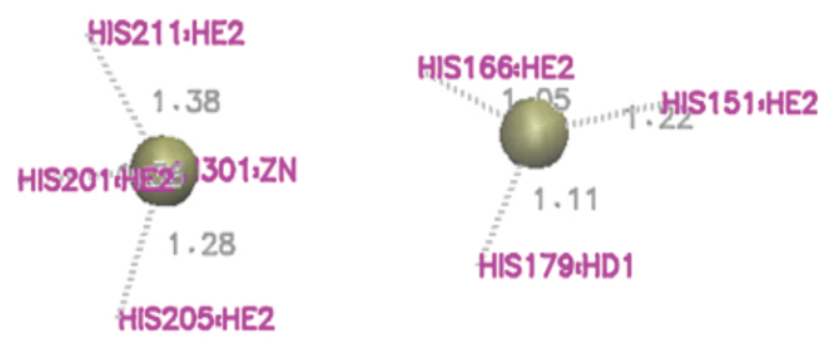

Fig 3: Interactions of catalytic $\mathrm{Zn}$ ions of MMP-3 with Astaxanthin 
Visualization of docked complex revealed that astaxanthin binds with the linker region of 1SU3 with binding affinity of $-10.5 \mathrm{kcl} / \mathrm{mol}$. Close interactions with VAL312, GLY72, GLN257, ARG262, GLY261, GLU311, LYS276, GLU113, ASP254 and ALA258 are shown in fig 4.A. Acidic amino acids ARG262 and GLU311 showed ionic interactions with oxygen atoms of ligand at distance of $3.90 \AA$ and $3.71 \AA$,

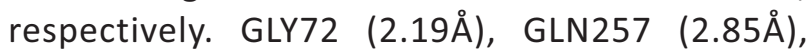

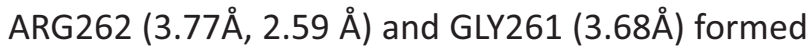
hydrogen bonds with oxygen and hydrogen atoms of ligand. Fifteen hydrophobic interactions were also observed with GLU311, LYS276, ASP254 and ALA258 (shown in Table 1; fig 4 B).

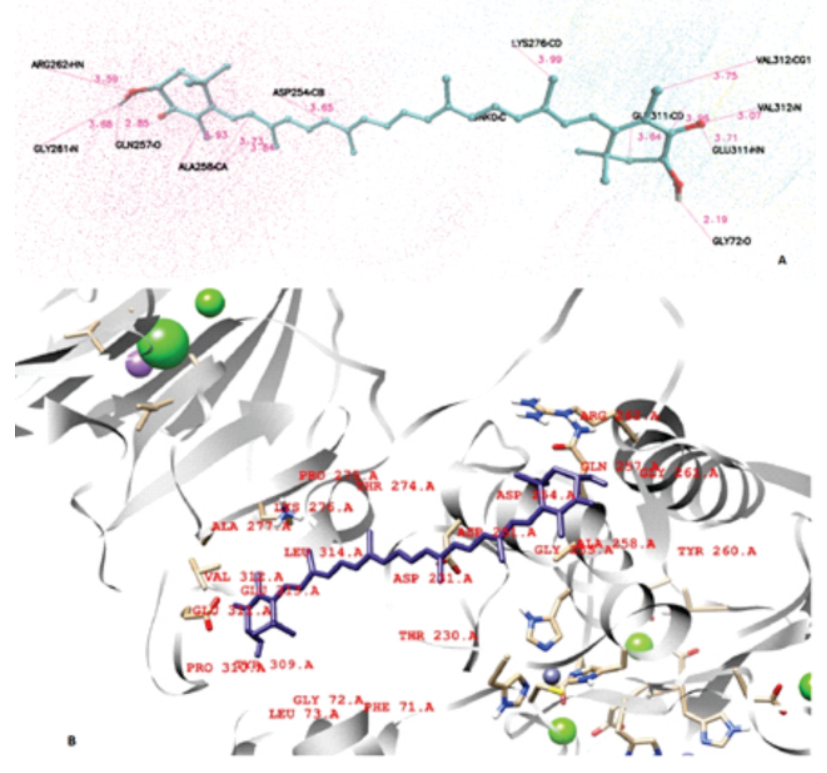

Fig 4 A: 2D depiction of Interstitial collagenase and astaxanthin docked pose. 4 B: 3D depiction of docked pose generated using Chimera.

Furthermore, to maintain the proper tertiary structure as well as the functional sites of MMP$1, \mathrm{Zn}^{2+}$ located at the catalytic site of the protein, showed several interactions with different histidine residues namely: HIS168, HIS183, HIS196, HIS281, HIS222 and HIS228 (Fig 5 and Table 1).

\section{Discussion}

Astaxanthina xanthophyll carotenoid, is a secondary metabolite naturally synthesized by a number of bacteria, microalgae, and yeasts. The commercial
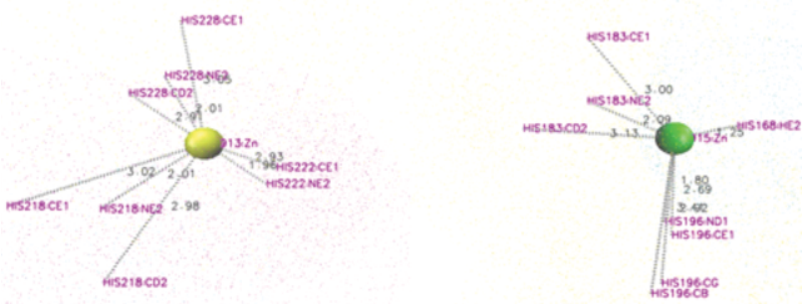

Fig 5: Interactions of catalytic $\mathrm{Zn}$ ions of MMP-1 with Astaxanthin

production of this pigment has traditionally been performed by chemical synthesis, but the microalga Haematococcus pluvialis appears to be the most promising source for its industrial biological production. Due to its collective diverse functions in skin biology, there is mounting evidence that astaxanthin possesses various health benefits and important nutraceutical applications in the field of dermatology. Although still unclear, a range of potential mechanisms have been hypothesized, through which astaxanthin can exert its benefits on skin homeostasis, including photo protective, antioxidant, and anti-inflammatory effects. ${ }^{23,24}$ Taking that into account, docking studies were conducted to evaluate the antioxidant activity of astaxanthin against MMPs.

Molecular docking protocols provided insight into the protein interaction and type of inhibition. ${ }^{25}$ Critical analysis of the docked complex revealed important interactions between the active residues and selected inhibitor astaxanthin for current study. Astaxanthin, like other ring structure inhibitors, binds to the His- $\mathrm{Zn}^{+2}$ active catalytic site of MMP proteinases resulting in down regulation or inhibition of collagenases and stromelysin. Due to its ring structure and keto-groups on both side it is considered a strong inhibitor to collagenase and acts as potent antioxidant thereby preventing the skin aging, inflammation and photo carcinogenesis. ${ }^{26}$

It is one of the most commercialized product used in nutraceutics, topical creams and sunscreen. The hydrophobic nature of the compound makes it a suitable candidate to prevent the water loss from skin. This compound due to its polar end groups prevents different skin protein from dryness, UV damage and maintains the structure of proteins. The hydrophobic and hydrophilic interactions of the compound with these metalloproteinase make 


\begin{tabular}{|c|c|c|c|c|}
\hline \multirow{2}{*}{$\begin{array}{l}\text { Complex } \\
\text { Stromelysin } 1 \text { (MMP-3)- } \\
\text { Astaxanthin }\end{array}$} & \multicolumn{2}{|c|}{ Hydrophobic Interactions } & \multicolumn{2}{|c|}{ Hydrogen Bond Interactions } \\
\hline & Residues & $\begin{array}{c}\text { Distance } \\
\text { (Å) }\end{array}$ & Residues & $\begin{array}{c}\text { Distance } \\
\text { (Å) }\end{array}$ \\
\hline & HIS201:CD2---UNK:C & 3.93 & HIS211:HE2---ZN301: ZN & 1.38 \\
\hline & HIS211:CE1---UNK:C & 3.97 & HIS205:HE2---ZN301: ZN & 1.28 \\
\hline & HIS211:CE1---UNK:C & 3.85 & HIS201:HE2---ZN301: ZN & 1.35 \\
\hline & SER225: CB---UNK:C & 3.93 & HIS151: HE2---ZN302: ZN & 1.22 \\
\hline & PHE86:CE1---UNK:C & 3.51 & HIS166: HE2---ZN302: ZN & 1.05 \\
\hline & PHE86:CZ---UNK:C & 3.94 & HIS179:HD1---ZN302: ZN & 1.11 \\
\hline & TYR223: CB---UNK:C & 3.46 & & \\
\hline & TYR223: CB---UNK:C & 3.98 & & \\
\hline & HIS205:CG---UNK:C & 3.94 & & \\
\hline & ASN162:C---UNK:C & 3.90 & & \\
\hline & LEU164:CD1---UNK:C & 3.60 & & \\
\hline & LEU164:CD1---UNK:C & 3.82 & & \\
\hline \multirow{29}{*}{$\begin{array}{l}\text { Interstitial collagenase } \\
\text { (MMP-1) -Astaxanthin }\end{array}$} & GLU311:CD---UNK:C & 3.64 & GLY72: O---UNK:H & 2.19 \\
\hline & LYS276:CD---UNK:C & 3.99 & GLN257: O---UNK:H & 2.85 \\
\hline & LYS276:CG---UNK:C & 3.27 & GLN257: O--- UNK:H & 3.77 \\
\hline & LYS276: CB---UNK:C & 3.94 & ARG262: N---UNK:H & 2.59 \\
\hline & VAL312:CG---UNK:C & 3.75 & ARG262: NH---UNK: O & 3.68 \\
\hline & GLU311: CB---UNK:C & 3.96 & GLY261: N---UNK:H & 3.91 \\
\hline & ASP254: CB---UNK:C & 3.67 & ARG262: N---UNK: O & 3.71 \\
\hline & ASP254: CB---UNK:C & 3.98 & GLU311:HN---UNK: O & 3.56 \\
\hline & ASP254: CB---UNK:C & 3.63 & HIS168:HE2--- ZN302:ZN & 1.25 \\
\hline & ALA258: CB---UNK:C & 3.73 & HIS183:NE2--- ZN302:ZN & 2.08 \\
\hline & ALA258: CB---UNK:C & 3.63 & HIS183:CE1-- ZN302:ZN & 3.00 \\
\hline & ALA258: CB---UNK:C & 3.84 & HIS196:ND1--- ZN302:ZN & 1.80 \\
\hline & ALA258: CB---UNK:C & 3.42 & HIS196: CB--- ZN302:ZN & 3.41 \\
\hline & ALA258:CA---UNK:C & 3.93 & HIS196:CG--- ZN302:ZN & 2.92 \\
\hline & ALA258:CA---UNK:C & 3.82 & HIS196:CE1--- ZN302:ZN & 2.69 \\
\hline & ALA258:CA---UNK:C & 3.87 & HIS183:CD2---ZN302:ZN & 3.13 \\
\hline & ALA258:CA---UNK:C & 3.85 & HIS222:NE2--- ZN302:ZN & 1.98 \\
\hline & & & HIS218:NE2--- ZN302:ZN & 2.01 \\
\hline & & & HIS228:NE--- ZN302:ZN & 2.01 \\
\hline & & & HIS228:CE1--- ZN302:ZN & 3.05 \\
\hline & & & HIS183:CD2---ZN302:ZN & 2.91 \\
\hline & & & HIS222:NE2--- ZN302:ZN & 2.98 \\
\hline & & & HIS218:NE2--- ZN302:ZN & 2.93 \\
\hline & & & HIS228:NE--- ZN302:ZN & 3.02 \\
\hline & & & HIS228:CE1--- ZN302:ZN & \\
\hline & & & HIS228:CD2--- ZN302:ZN & \\
\hline & & & HIS218: CD2--- ZN302:ZN & \\
\hline & & & HIS222:CE1--- ZN302:ZN & \\
\hline & & & HIS218:CE1--- ZN302:ZN & \\
\hline
\end{tabular}

astaxanthin a suitable candidate for future use to prevent premature aging and other pathological conditions due to stress.

\section{Conclusion}

This study has led to a better understanding of astaxanthin interaction with the MPPs and opens new avenues for the future research in nutraceutics and antioxidants. We concluded that astaxanthin is a strong inhibitor for MMPs and can bind to the his-zin catalytic site of proteinases which in turn prevent the disruption of different skin proteins. Moreover, a strong hydrophobic and hydrophilic interaction also been observed which help to maintain the skin membrane integrity thereby preventing skin damage and other pathological conditions.

\section{REFERENCES}

1. Kuhn R, Soerensen NA. The coloring matters of the lobster (Astacusgammarus L.) Z Angew Chem. 1938; 5: 465-6.

2. Nootem J, Chalorak P, Meemon K, Mingvanish W, Pratumyot $\mathrm{K}$, Ruckthong $\mathrm{L}$, et al. Electrospun cellulose acetate doped with astaxanthin derivatives from Haematococcuspluvialis 
for in vivo anti-aging activity. RSC advances. 2018; 8: 37151 8.

3. Wang HM, Wu PF. 7-Hydroxydehydronuciferine induces human melanoma A375. S2 cell death via autophagy and apoptosis in vitro and in vivo investigations. Toxicol Lett. 2013; S87.

4. Wu SYS, Wang HMD, Wen YS, Liu W, Li PH, Chiu CC, et al. 4(Phenylsulfanyl) butan-2-One Suppresses Melanin Synthesis and Melanosome Maturation In Vitro and In Vivo. Int J Mol Sci. 2015; 16: 20240-57.

5. Fisher GJ, Datta SC, Talwar HS, Wang ZQ, Varani J, Kang S, et al. Molecular basis of sun-induced premature skin ageing and retinoid antagonism. Nature. 1996; 379: 335.

6. Das RN, Balupillai A, David E, Santhoshkumar M, Muruhan S. Naringin. A Natural Flavonoid, Modulates UVB RadiationInduced DNA Damage and Photoaging by Modulating NER Repair and MMPS Expression in Mouse Embryonic Fibroblast Cells. Journal of Environmental Pathology, Toxicology and Oncology. 2020; 39: 191-9.

7. Leirós GJ, Kusinsky AG, Balañá ME, Hagelin K. Triolein reduces MMP-1 upregulation in dermal fibroblasts generated by ROS production in UVB-irradiated keratinocytes. Journal of dermatological science. 2017; 85: 124-30.

8. Tominaga $\mathrm{K}$, Hongo N, Fujishita $\mathrm{M}$, Takahashi $\mathrm{Y}$, Adachi $\mathrm{Y}$. Protective effects of astaxanthin on skin deterioration. J Clin Bio chem Nutr. 2017; 61: 17-35.

9. Dong KK, Damaghi N, Picart SD, Markova NG, Obayashi K, Okano Y, et al. UV-induced DNA damage initiates release of MMP-1 in human skin. Experimental dermatology. 2008; 17: 1037-44.

10. Chen CY, Cheng KC, Chang AY, Lin YT, Hseu YC, Wang HM. 10Shogaol, an antioxidant from Zingiberofficinale for skin cell proliferation and migration enhancer. Int. J. Mol. Sci. 2012; 13: 1762-77.

11. Wlaschek M, Wenk J, Brenneisen P, Briviba K, Schwarz A Sies $H$, et al. Singlet oxygen is an early intermediate in cytokine-dependent ultraviolet-A induction of interstitial collagenase in human dermal fibroblasts in vitro. FEBS Lett. 1997; 413: 239-42.

12. Yuan JP, Peng J, Yin K, Wang JH. Potential health-promoting effects of astaxanthin: A high-value carotenoid mostly from microalgae. Mol Nutr Food Res. 2010; 55: 150- 65.

13. Sajjad W, Ahmad M, Khan S, Ilyas S, Hasan F, Celik C, et al. Radio-protective and antioxidative activities of astaxanthin from newly isolated radio-resistant bacterium Deinococcus sp. strain WMA-LM9. Annals of Microbiology. 2017; 67: 443-55.
14. Mortensen A, Skibsted L, Sampson J, Rice-Evance C, Everett SA. Comparative mechanisms and rates of free radical scavenging by carotenoid antioxidants. FEBS Lett. 1997; 418: 91-7.

15. Morris GM, Huey R, Lindstrom W, Sanner MF, Belew RK, Goodsell DS, et al. AutoDock4 and AutoDockTools4: automated docking with selective receptor flexibility. J Comput Chem. 2009; 30: 2785-91.

16. Istyastono EP, Radifar M, Yuniarti N, Prasasty VD, Mungkasi S. PyPLIF HIPPOS: A Molecular Interaction Fingerprinting Tool for Docking Results of AutoDockVina and PLANTS. Journal of Chemical Information and Modeling. 2020; 60: 3697-702.

17. Pettersen EF, Goddard TD, Huang CC, Couch GS, Greenblatt DM, Meng EC, et al. UCSF Chimera--a visualization system for exploratory research and analysis. J Comput Chem. 2004; 25: 1605-12.

18. Humphrey W, Dalke A, SchultenK. VMD - Visual Molecular Dynamics. J Mol Graph. 1996; 14: 33-8.

19. Pittayapruek P, Meephansan J, Prapapan O, Komine M, Ohtsuki M. Role of Matrix Metalloproteinases in Photoaging and Photocarcinogenesis. Int J Mol Sci. 2016; 17: 868.

20. Stote RH, Karplus M. Zinc binding in proteins and solution: a simple but accurate nonbonded representation. Proteins. 1995; 23:12-31.

21. Handoko SD, Ouyang X, Su CT, Kwoh CK, Ong YS. QuickVina: accelerating AutoDockVina using gradient-based heuristics for global optimization. IEEE/ACM Trans Comput Biol Bioinform. 2012; 9: 1266-72.

22. Tatiana V, Sérgio S. Comparing AutoDock and Vina in Ligand/Decoy Discrimination for Virtual Screening. Appl Sci. 2019; 9: 4538.

23. Zuluaga $M$, Barzegari A, Letourneur D, Gueguen V, PavonDjavid G. Oxidative stress regulation on endothelial cells by hydrophilic astaxanthin complex: chemical, biological, and molecular antioxidant activity evaluation. Oxid Med Cell Longev. 2017.

24. Davinelli S, Nielsen ME, Scapagnini G. Astaxanthin in Skin Health, Repair, and Disease: A Comprehensive Review. Nutrients. 2018; 10: 522.

25. Sali A, Blundell T. Comparative protein modeling by satisfaction of spatial restraints. J Mol Biol. 1993; 234: 779-815.

26. Jozic D, Bourenkov G, Lim NH, Visse R, Nagase H, Bode W, et al. X-ray Structure of Human proMMP-1 new insights into procollagenase activation and collagen binding. J Biol Chem. 2005; 280: 9578-95. 